\title{
Sensing bone mass
}

A new study published in Nature Communications provides a feedback mechanism to explain how sensory innervation of the bones is required for maintenance of optimal bone mass. "Sensory nerves can detect bone density changes by responding to the concentration of prostaglandin $\mathrm{E}_{2}$ $\left(\mathrm{PGE}_{2}\right)$ in the bones," says $\mathrm{Xu} \mathrm{CaO}$, corresponding author.

$\mathrm{PGE}_{2}$, which is produced from arachidonic acid by the enzymatic activity of cyclooxygenase 2 (COX2) and is widely known for its function in inflammatory pain, was previously thought to regulate bone turnover by directly affecting osteoblasts via the $\mathrm{PGE}_{2}$ receptor EP4 subtype (EP4). However, the researchers found no effect on bone turnover when they deleted this receptor specifically from osteoblasts in mice. By contrast, mice lost bone mass when EP4 was deleted from the sensory nerves.

This same bone loss phenotype was achieved in osteoblast-specific COX2-knockout mice, indicating that osteoblast production of COX2 controls expression of $\mathrm{PGE}_{2}$, which might in turn sensitize sensory neurons.

Sympathetic tone is known to favour bone loss and CREB signalling can reduce this sympathetic activity, so any signal that drives CREB activity in the sensory nerves can loop back to maintain bone mass. Indeed, unlike in wild-type mice, the researchers show that CREB signalling in the hypothalamus of sensory nervespecific EP4-knockout mice was unresponsive to $\mathrm{PGE}_{2}$ injection.

To provide further evidence of this centrally processed homeostatic mechanism, the researchers injected the $\beta$-adrenergic antagonist (to inhibit sympathetic activity) propranolol into the mice with bone loss phenotypes (that is, sensorynerve-specific EP4-knockout mice and osteoblast-specific COX2knockout mice). In both groups, injection with propranolol reduced the degree of bone loss.

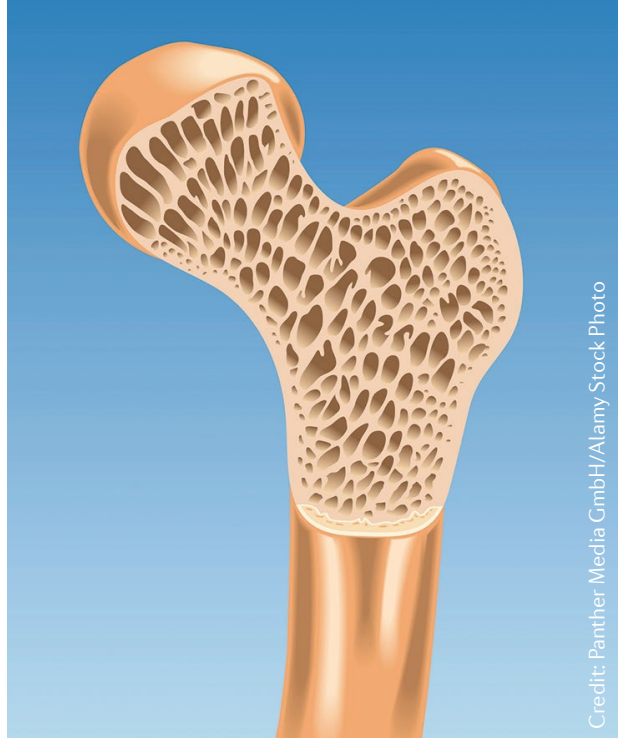

Another therapeutic strategy tested was to increase levels of $\mathrm{PGE}_{2}$ in the bones of mice; injection of a small-molecule inhibitor of the $\mathrm{PGE}_{2}$-degrading enzyme PGDH (SW033291) boosted the regeneration of trabecular bone in surgically induced defects.

Together these findings

mice lost

bone mass when EP4 was deleted from the sensory nerves 5) provide a neuro-bone crosstalk mechanism for bone homeostasis and provide new strategies to regenerate bone.

Nicholas J. Bernard

ORIGINAL ARTICLE Chen, $\mathrm{H}$. et al. Prostaglandin E2 mediates sensory nerve regulation of bone homeostasis. Nat. Commun. 10, 181 (2019)

\section{Calculating TNF dynamics}

A new study published in Science Translational Medicine shows that high concentrations of circulating TNF after adalimumab (TNF inhibitor) therapy are representative of high levels of biologically inactive TNF in complex with the inhibitor.

TNF inhibitors are the most widely prescribed biologic DMARDs for rheumatic diseases, particularly for rheumatoid arthritis (RA). Counterintuitively, concentrations of circulating TNF have previously been noted to increase shortly after beginning TNF inhibitor therapy, which is thought to result from the TNF inhibitor interfering with standard TNF detection methods.

This confusion, as well as the complexity in the dynamics of circulating TNF, has made it difficult to calculate the association between TNF levels and RA disease and remission, or to make clinical decisions about when a patient should cease TNF inhibitor therapy.
To study the dynamics of TNF in the blood, the researchers developed an ELISA that could be used to detect low levels of TNF and that could discriminate between free TNF and TNF-adalimumab complexes. Using this method in combination with chromatography, the researchers confirmed that there is a sharp rise in blood TNF concentration in patients with RA 4 weeks after beginning adalimumab therapy. A similar effect occurred in healthy volunteers treated with a single dose of adalimumab. The majority of TNF circulating in patients with RA was in complex with adalimumab and was biologically inactive. Furthermore, the TNF concentration remained high over the course of a 1-year follow-up.

Despite substantial inter-patient variation in TNF levels, the researchers noted little intra-patient

variation between 4-weeks and 1-year follow-up,

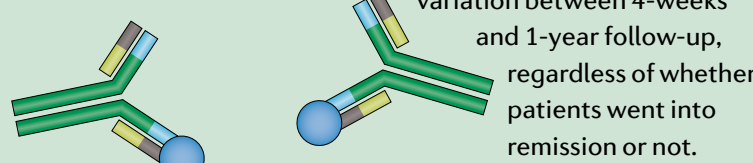

By contrast, a strong association was noted between low levels of TNF in the first 4 weeks of adalimumab therapy and the later emergence of anti-drug antibodies. Furthermore, patients with low levels of TNF in these early stages of therapy were less likely than other patients to enter remission within a year of therapy.

Together these data show that measuring total TNF concentration in the blood is not useful as a biomarker to decide whether or not to continue TNF inhibitor therapy, but that measuring TNF levels in the first few weeks of starting TNF inhibitor therapy might predict the chances of remission.

$\begin{array}{ll}\text { a similar } & \text { the first } \\ \text { effect } & \text { inhibitor therapy might predict the } \\ \text { chances of remission. }\end{array}$

occurred

in healthy volunteers

ORIGINAL ARTICLE Berkhout, L. C. et al. Dynamics of circulating TNF during adalimumab treatment using a drug-tolerant TNF assay. Sci. Transl. Med. 11, eaat3356 (2019) 